\title{
Review Paper: The Social Vulnerability of Older People to Natural Disasters: An Integrative Review
}

\author{
Leila Daddoust $^{1 *}$ (D), Hamid Reza Khankeh² (D, Abbas Ebadi ${ }^{3}$ (D), Robab Sahaf ${ }^{4,5}$ (D), Maryam Nakhaei ${ }^{6}$ (D), Ali Asgary ${ }^{7}$ (C) \\ 1. Research Center in Emergency \& Disaster Health, University of Social Welfare and Rehabilitation Sciences, Tehran, Iran. \\ 2. Department of Clinical Science and Education, Karolinska Institute, Stockholm, Sweden. \\ 3. Department of Nursing, Faculty of Nursing, Baqiyatallah University of Medical Sciences, Tehran, Iran. \\ 4. Iranian Research Center on Ageing, Faculty of Ageing, University of Social Welfare and Rehabilitation Sciences, Tehran, Iran. \\ 5. Research Center of Social Determinants of Health, University of Social Welfare and Rehabilitation Sciences, Tehran, Iran. \\ 6. Department of Nursing, School of Nursing and Midwifery, Birjand University of Medical Sciences, Birjand, Iran. \\ 7. Advanced Disaster, Emergency and Rapid-response Simulation, York University, Toronto, Canada.
}

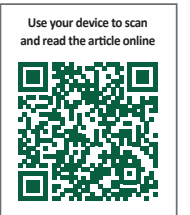

Citation: Daddoust L, Khankeh HR, Ebadi A, Sahaf R, Nakhaei M, Asgary A. The Social Vulnerability of Older People to Natural Disasters: An Integrative Review. Health in Emergencies and Disasters Quarterly. 2018; 4(1):5-14. http://dx.doi.org/10.32598/ hdq.4.1.5

http://dx.doi.org/10.32598/hdq.4.1.5

Funding: See Page 12

\section{(c) (i) (8)}

Article info:

Received: 20 Apr 2018

Accepted: 28 Aug 2018

Available Online: 01 Oct 2018

\section{Keywords:}

Elderly, Social vulnerability, Natural disasters, Integrative review

\section{A B STRACT}

Background: Determining social vulnerability of elderly people is a prerequisite for risk analysis in natural disasters. Understanding and comprehension of different dimensions of elderly social vulnerability by using international experiences is a must to determine and ultimately measure this phenomenon. In this study, we tried to understand the dimensions of elderly social vulnerability in natural disasters based on international experiences.

Materials and Methods: An integrative review of literature was conducted to clarify the elderly social vulnerability concept in natural disasters. We used keywords such as "elderly", "social vulnerability", and "natural disasters" in texts and titles in electronic databases of Medlib Iran Medex, Magiran, SID, Irandoc, Web of Science, Google Scholar CINAHL, ProQuest, Ovid, Ageline, Scopus, Embase, and Pub Med. At last 25 articles were found issued from 1986 to 2018. Their inclusion criteria were written in Persian or English language and had search keywords in their texts, titles, or and keywords. Commentary or editor letters were excluded. Each article was examined according to subjects, definition of social vulnerability, theoretical aspects, outcomes and antecedents of social vulnerability. For analyzing, controversial content analyses was used.

Results: The results of the concept analysis showed that social vulnerability is a complex, dynamic, challenging, multi-dimensional and pre-existing condition that is dependent on individual characteristics as well as economic status. It is influenced by the culture and elderly people's place of living.

Conclusion: Reducing social vulnerability of older adults in natural disasters is not possible without paying enough attention to this issue and increasing their empowerment. Therefore, extensive research to identify this concept in related contexts and carry out qualitative research will be very effective in defining this concept and providing the appropriate tools for its measurement.

\footnotetext{
* Corresponding Author:

Leila Daddoust, PhD Candidate

Address: Research Center in Emergency \& Disaster Health, University of Social Welfare and Rehabilitation Sciences, Tehran, Iran

E-mail: daddoust@yahoo.com
} 


\section{Introduction}

ased on the statistics, nearly one billion people in the world are directly and indiB rectly involved in disasters [1]. Of these, the death of elderly people is more than expected [2]. According to official reports, Asia has encountered more disasters than other continents [3]. Because of its geographical location, Iran is considered one of the most disaster-prone countries, especially natural disasters in Asia and the world [4]. Demographically, the aging process in Iran is also increasing [5]; the proportion of older people in this country has increased from $7.22 \%$ in 2006 to $8.20 \%$ in $2011[5,6]$. It is predicted that in 2050 , the older people will comprise more than $20 \%$ of Iran's population [7].

At the time of natural disasters, older people compose a large part of casualties [2]. The occurrence of natural disasters is a challenging and frustrating problem for older people, and they experience the deprivation in various resources [8]. Although social vulnerability is neither age-dependent nor age-related, the elderly are at greater risk than others owning to their specific situation [9]. The social vulnerability is a part of assessment and analysis of natural hazards and provides vital information for risk assessment and reduction [6]. It occurs when the inequality in facing hazardous coincides with inequality in access to resources [10].

An individual's vulnerability includes a series of vulnerabilities related to the social levels that the individual belongs to [11]. In other words, it relies on the conditions of a specific region in terms of risk, exposure, preparedness, prevention, and response to particular risks [12]. Social vulnerability is a product of social inequality and a dynamic social issue [6]. It is "the characteristics of a person or group and their situation that influence their capacity to anticipate, cope with, resist, and recover from the impact of a natural hazard" [13].

Studies indicate that in order to have higher risk reduction and preparedness against natural hazards, in the first step, it is necessary to identify and assess the types of vulnerabilities, and in the next step, to measure those using appropriate tools [14]. In the latest document of the World Conference on Disaster Reduction, the Hyogo Framework for Action 2010-2015, and Sendai Framework for Disaster Risk Reduction 2015-2030 (SFDRR), vulnerability measurement is listed as one of the key activities in disaster management $[6,15]$. Although everybody agrees on the dynamics and multi-dimensionality of social vulnerability, nobody gives a clear and applicable definition because of the complex nature of social systems and the increasing growth of research and development in this area [6].

Obviously the ability to measure social vulnerability is the preliminary step for limiting its root causes and consequences [16]. Likewise, decision makers and health authorities should assess, identify and eliminate the vulnerability causes and factors to promote the health of older people and reduce injuries and casualties in this vulnerable group in natural disasters. This integrative review study aimed at understanding the various dimensions of social vulnerability in elderly people based on international experiences and research studies.

\section{Materials and Methods}

The present study is an evidence-based integrative review conducted based on Broome's method (2000) [17] in the following three steps:

\section{Literature search}

At this stage, the exact definition of literature search strategies is presented to increase the reputation of the review.

\section{Data evaluation}

Research team evaluate the accuracy of data and their relevance with the study aim and, most importantly, the quality of extracted literature to provide first-hand information.

\section{Data analysis}

Analysis of data requires that the data extracted from the primary sources be sorted, categorized and summarized into a unified and integrated conclusion. An uncomplicated and unambiguous interpretation of the primary sources, along with an accurate combination of evidence, are the goals of the data analysis step. Extracted data are compared item by item so that similar data are categorized and grouped together. Subsequently, these coded categories are compared which further the analysis and synthesis process (Figure 1). The method consists of data reduction, data display, data comparison, conclusion drawing, and verification $[17,18]$.

As the first stage, extensive search in health literature were performed using keywords: "social vulnerability", "elderly", "natural disasters," and "natural hazards". The used online databases were MedLib, IranMedex, MagIran, SID, IranDoc, Web of Science, Google Scholar, 
CINAHL, PubMed, Embase, ProQuest, Ovid, AgeLine, and Scopus. Some sources were also looked at using snowball sampling by checking the reference list of online published papers. The inclusion criteria were a variety of qualitative and quantitative studies in English or Persian, the existence of the mentioned keywords in the title or abstract of papers, and publication in prestigious foreign and domestic journals. Letter to the editors and one-page proposals were excluded from the study. In total, according to the inclusion criteria, 25 published articles were selected dated from 1986 to 2018.

At the second step, in order to analyze and conceptualize the social vulnerability of elderly people to natural disasters, we tried to answer following questions: 1 . What are the features of social vulnerability to natural disasters in elderly people?; 2 . How can this concept be clearly defined?; 3 . How to measure the social vulnerability of older people to natural disasters?
Finally, the process of data reduction, data display, data comparison, and conclusion were performed on the papers selected using Broome's approach in MAXQDA software. During the data collection, there was no interpretation and the papers' own phrases were used. The validity of the data was verified by the three researchers and the discussion was arranged in case of disagreement on some issues.

\section{Results}

In this section, we present the results of examination, evaluation, and analysis of extracted data related to social vulnerability of elderly people to natural disasters.

Table 1 presents an example of data reduction as the first step of data analysis based on the designed questions, on one of the features of the social vulnerability concept (data display stage). The social vulnerability
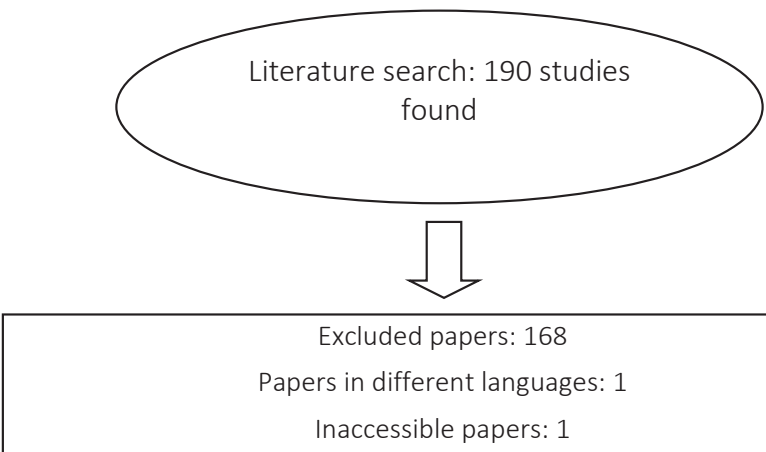

Excluded papers after checking the title and purpose: 127

Excluded papers after checking the abstract: 39

Excluded papers after reviewing the full text: 22

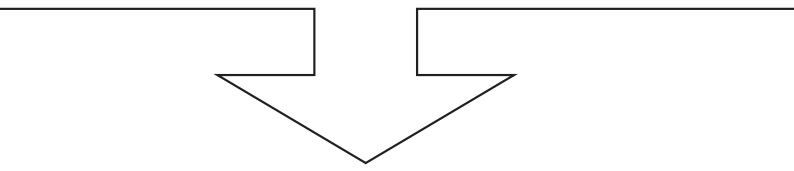

Selected studies: 22

Added studies after checking the

reference list of selected studies: 3

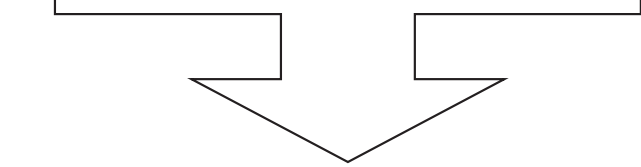

Total reviewed studies: 25

Figure 1. The process of selecting studies for review 
concept of elderly people to natural disasters had five themes or features: complex/hidden, dynamic and challenging, multidimensional, revealed, and pre-existing condition (contextual); four themes: individual characteristics, financial situation, culture, and place of residence as factors affecting social vulnerability; and three themes: health problems, limitations, and financial problems as the consequences of social vulnerability. Based on these themes, a theoretical definition is presented.

\section{Conceptualization of social vulnerability to natu- ral disasters in older people}

Social vulnerability as a dynamic and challenging concept

The social vulnerability is lacking from a clear and practical definition due to the complex nature of social systems, and increased research and development in this area; however, since it is a dynamic and challenging concept with a diverse academic discourse [19], its definition is clear. Adger discussed three characteristics of vulnerability and vulnerability research that present particular problems when devising vulnerability indicators: complexity and limited understanding of the concept, the issue of different scales, and the dynamism [20]. This concept is dynamic and depends on the scale, i.e. its characteristics and drivers change over time [21].

\section{Social vulnerability as a multidimensional concept}

Vulnerability denotes the physical, economic, political or social sensitivity of a community to damage against a destabilizing phenomenon with a human or natural origin [22], and it has a distinctly multifaceted nature, including issues related to livelihoods, place, security and gender [23]. In other words, this concept reflects poverty, race, ethnicity, gender, and age [6].

\section{Social vulnerability as a contextual concept}

In their study, Cutter et al. concluded that the social vulnerability is independent of the type of hazards or source of threats [24]. Otherwise speaking, social vulnerability "is a pre-existing condition or an inherent property of existing communities, irrespective of the natural hazard of interest". This feature is key to the vulnerability, and its extent and range make the results of a natural disaster unique [25]. This concept refers to the characteristics of communities such as urbanization, economic growth, and economic prosperity. The vulnerability experience depends on specific hazard (context), characteristics of the person, and characteristics of the situation [22].

\section{Social vulnerability as a complex/hidden concept}

Social vulnerability "is often hidden, complex and nested in various human aspects and contingencies bound to different levels of society" $[19,22]$. According to Mavhura et al., our understanding of social vulnerability is hidden in dynamic contextual conditions and is revealed after disasters [21], when specific demographic groups such as the poor, the elderly, and minorities show different patterns of pain and suffering [26].

Social vulnerability as a concept derived from demographic factors

Social vulnerability "has a complex characteristic produced by a combination of factors derived especially (but not entirely) from class, gender and ethnicity" [22]. According to Singh et al. at the community level, some people are more vulnerable than others to haz-

Table 1. An example of how the semantic units related to one of the features of social vulnerability to natural disasters in older people were integrated and categorized

\begin{tabular}{|c|c|c|c|}
\hline Features & Subcategory & Codes & Semantic Unit \\
\hline & Physical and mental challenges & $\begin{array}{l}\text { Social injustice, inequality of } \\
\text { risk, unequal access to services } \\
\text { and resources }\end{array}$ & $\begin{array}{l}\text { There are many physical and psycho- } \\
\text { logical problems that elderly people } \\
\text { are unable to manage; so the neces- } \\
\text { sary interventions to address injustice, } \\
\text { unequal risk, lack of access to resources } \\
\text { and services are needed [19]. }\end{array}$ \\
\hline Individual limitations & Education & $\begin{array}{l}\text { Literacy as a barrier to receiv- } \\
\text { ing services, Literacy as a } \\
\text { reason for having trouble } \\
\text { accessing to resources, Inability } \\
\text { to receive services because of } \\
\text { low educational level }\end{array}$ & $\begin{array}{l}\text { Education is a variable dependent on } \\
\text { the socioeconomic status. People with } \\
\text { higher education usually have more } \\
\text { money and savings. The low level of } \\
\text { education even reduces one's ability } \\
\text { to get the early warnings and access } \\
\text { to information related to the time of } \\
\text { recovery [20]. }\end{array}$ \\
\hline
\end{tabular}


ards, loss of property, and pain. Key characteristics of these variations of impact include class, caste, ethnicity, gender, disability, and age [22, 27]. These characteristics together cause vulnerability and exacerbate the problem and its consequences [28].

\section{Factors affecting the social vulnerability to natural disasters in older people}

The first factor that affects the social vulnerability of older people is "individual characteristics". The individual characteristics have a greater impact on the effects of natural disasters and increase the vulnerability. Having chronic illness, vision problems, hearing and motor problems, loneliness, and dependence on children are characteristics that make a person more vulnerable at the old age. Elderly people, especially those with poor health or poor socioeconomic status, are more vulnerable to natural disasters, especially to those with rapid onset. The elderly's physical problems expose them to vulnerabilities during all stages of a disaster [8]. Extensive communications and strong social networks, by providing the necessary information, help people to be well prepared for disasters $[8,19,29]$.

Most elderly people live alone; "Living alone increases the risk of social isolation, which may, in turn, be associated with poorer mental and physical health, and leads to problems in escape and recovery from emergency situations" [6]. The influence of race and class on producing social inequalities has a long history. For example, in response to Hurricane Katrina, many ethnic and racial minorities in the US experienced high vulnerabilities because of social inequalities [24]. The effect of gender on social vulnerability is undeniable. Women are more vulnerable than men; "women are more likely than men to hold low-status jobs in the service industry, which often disappear after a disaster strikes" [30].

The second effective factor is "financial situation." Marshall Jr and Mathews [31] argued that, regarding the poor financial situation of the elderly in comparison with the general population, events such as natural disasters have more effects on older people. According to Chau et al. [6], living alone may be further exacerbated by financial difficulties (poverty) which can limit one's ability to access resources. Poor older people may not be able to afford fuel, food, clothing, or transportation.

The higher the percentage of poor older people, the higher would be the social vulnerability [6]. The concept of vulnerability is deeply rooted in natural hazards and poverty [32]. Lack of financial resources after a natural disaster make the elderly more vulnerable than others [33]. In general, poor people are more vulnerable than rich people; "Poor people have less money to spend on preventative measures, emergency supplies, and recovery efforts" [24]

The third factor is "place of residence". "Vulnerabilities vary geographically, over time, space and among different social groups" [21]. Therefore, it is necessary to understand the spatial effects of natural disasters and social conditions that lead to different outcomes [22]. According to Cutter et al. [24], poor people are more likely to live in substandard housing, which can be a major disadvantage when disasters occur, and during disasters, are less likely to have access to critical resources and lifelines, such as communications and transportation.

The final influential factor is "culture". Culture and its components can improve or reduce vulnerability and its various dimensions. For this reason, understanding of disaster based on cultural background is necessary and evident [34]. Indigenous beliefs, customs, and lifestyles refer to the cultural aspect [19]. In this regard, results of Jogia et al. [35] showed that culture and its components not only do not impede the disaster risk reduction, but also play an important role as a facilitator in reducing disaster risk. However, sometimes it can increase the vulnerability. That is why those plans that are sensitive to the cultural differences of communities affected by natural disasters, are very effective in preventing, preparing, and evaluating vulnerabilities of the people [35].

\section{Consequences of social vulnerability to natural disasters in older people}

The first consequence of the elderly's social vulnerability to natural disasters is "health problems". At the time of disasters, the situation of older people with chronic illness are exacerbated due to the lack of access to medications or treatment follow-up. In such situations, they are unable to escape, respond quickly or evacuate, and suffer injury and major problems. In addition, because of losing their home or children, they are physically, mentally and emotionally disturbed, and become unable to care for themselves and hence, their previous problems are exacerbated [8]. According to Fernandez et al. [36], in sudden onset disasters such as earthquake and flooding, the elderly may not be physically able to get ready or evacuate buildings quickly. Moreover, in this situation, older people have more physical injuries compared to other age groups, and their mortality increases [37]. 
The second consequence is "incidence of limitations". The occurrence of disasters damages the properties and assets of people. Under these conditions, some lose their jobs, and unemployed people with bad financial standing cannot earn money. When this situation continues, it gets worse and people become more vulnerable. Physical, sensory (speech, hearing, and visual impairments) or cognitive impairments and chronic illnesses restrict physical function and activities of old people [6]. According to Chau et al. [6], since disability disrupt the elderly's independence and increase their dependency, it is a direct indicator of vulnerability in older people.

The final consequence is "financial implications". Unfortunately, the bad conditions of communities, neighborhoods, and poor people are painfully revealed after natural disasters. "Poverty is a risk factor when faced with a disaster" [9]. Because there is a high level of poverty among the elderly, it is reasonable to claim that older people have high vulnerability to disasters due to financial problems. "Poverty limits the older people's ability to acquire resources necessary to maintain their well-being" [6]. Chau et al. quoted that: "impoverished older people may not be able to afford fuel, food, or clothing to keep them warm in extremely cold weather or may have limited access to services and resources for post-disaster rehabilitation, including accommodation, clothing, or transportation" [6].

\section{Theoretical definition of social vulnerability to natural disasters in older people}

Summarizing all the results and themes obtained from the extraction, classification, and integration of data led to the formation of a final definition of the elderly's social vulnerability to natural disasters. Social vulnerability is a situation that depicts the capabilities and limitations of an elderly person's life in a contextual background. This situation, in various forms and realities, represents the social, economic, and cultural conditions of a community where the elderly lives. These conditions which are often associated with many limitations, although in normal circumstances is hidden amidst various aspects of life called "life difficulties" and is even acceptable, are revealed when the problems are uncontrollable and unmanageable.

When unfavorable physical conditions disrupt the activities of daily living; when the people lose the mental capacity to face and accept the new conditions and give up; when poverty makes people unable to manage their life's finances due to economic conditions after a natural disaster; or when people lose their place of residence and ends up in a new and unfamiliar place, the normal process of life disrupts. It threatens people's health, independence, and dignity, and creates a new perspective on pre-existing conditions which challenges people's ability to survive and cause them to face with many problems resulting from the new conditions.

In brief, the elderly's social vulnerability is a complex, dynamic, challenging, multidimensional, and contextual concept that depends on individual's characteristics and economic conditions and is influenced by the culture and the place of residence. This concept reveals itself more at the time of natural disasters and gives the elderly a different direction in life.

\section{Discussion}

The findings of our study suggest that social vulnerability is a challenging and dynamic concept, which can, at the same time, have many dimensions. In some cases, the social vulnerability of older people may be visible, and in other circumstances, hidden and can only be felt. In a general statement, vulnerability can be identified as a set of underlying conditions or problems in the context of individual and social life. Conceptually, providing a precise definition acceptable by all researchers and theorists is difficult, and this deficiency creates a major problem in designing tools for social vulnerability assessment.

Various and sometimes, contradictory definitions of vulnerability indicate that different scholars have looked at this issue and assessed it on the basis of different approaches. "The different definitions of vulnerability can also be viewed from a functional and subject- or objectoriented perspective, i.e. considering the end-user of the scientific assessment results" [38]. Paul believed that the concept of vulnerability is deeply rooted in natural hazards; "It is true that vulnerability has no universal definition, but undoubtedly it is a powerful analytical tool in describing the existing condition of susceptibility to harm, powerlessness, and marginality of both physical and socio-ecological systems" [32].

Alexander has defined vulnerability as "the potential for casualty, destruction, damage, disruption or other forms of loss with respect to a particular element" [36], while other scholars have different attitudes toward this concept, and have defined it as "the apparent weakness of the physical and social systems to particular hazards" [36], where the physical system vulnerability is defined "in terms of fragility curves for infrastructure and quantified as a function of hazard intensity", and social vul- 
nerability is "a complex function of social, economic, political, and cultural variables" [36].

Some believe that vulnerability varies depending on geographic location, demographic characteristics and hazards. The demographic characteristics of an area become important when it consists of vulnerable groups such as the elderly, children and the low income population [39]. However, vulnerability is "the incapacity of a community to absorb, via auto-adjustments, the impacts of a change in the environment" [36]. Put it differently, social vulnerability is the incapacity of community to adapt to such changes. Singh et al. [22] quoted that social vulnerability is a product of social inequalities and is a social dynamic issue rooted in gender, class, race, culture, nationality, age, and other power relationships.

"It is defined as the susceptibility of social groups to the impacts of hazards, as well as their resiliency, or ability to adequately recover from them" [22]. A review of the literature reveals no single conceptual framework for social vulnerability. Over the past few decades, different conceptual models have been presented with the goal of developing vulnerability assessment methods. The following are the most common models:

\section{Two-layer structure of vulnerability}

Chamber suggested that vulnerability concept has external and internal sides. The external side includes risk management, response to disaster, and social inequalities. Vulnerability within the framework of hazard and risk: This model considers disaster risk as the sum of hazard, exposure, vulnerability, and capacity measures; where hazard is shown by probability and severity; exposure is characterized by structure, population and economy; vulnerability has physical, social, economic and environmental dimensions; and capacity includes physical planning and managerial, social, and economic capacities [39].

\section{Vulnerability in the global/environmental change community}

In this framework, vulnerability includes exposure, sensitivity, and resilience. Exposure contains a set of threatened elements such as individuals, households, states, ecosystem, and so on subjected to damage and characteristics of the threat (frequency, magnitude, and duration). The sensitivity is determined by the human (social capital and endowments) and environmental conditions (natural capital or biophysical endowments) of the system. Resilience is determined by adjustments and adaptation. Vulnerability of a system to hazards is linked to the different human and biophysical conditions, processes operating on human- environment system, disturbances emerged from these conditions, and exposure and responses [36].

\section{Pressure and release model}

In this model, vulnerability has three layers of causes that are linked hierarchically. Wisner et al. called the combination of these layers a "chain of causation" [27]; "It is a sequence of factors and processes that leads us from the disaster event and its proximate causes back to ever more distant factors and processes that initially may seem to have little to do with causing the disaster" [27]. Root causes are distant causes of disaster, and unsafe conditions are directly linked to disaster. Between them are dynamic pressures that act as a medium for the progression from root causes to unsafe conditions [27].

\section{Access model}

This model is a dynamic model unlike "pressure and release" model which is static. It operates on a microlevel but considers complex processes at the local, national, and international levels. "This focuses on the unsafe conditions arise in relation to the economic and political processes that allocate assets, income and other resources in a society" [27], and explains its interaction with hazard.

\section{A holistic approach to risk and vulnerability}

This approach focuses on the importance of assessing vulnerability from a comprehensive and multidisciplinary point of view. It pays attention to the consequences of direct physical impacts (exposure to risk and susceptibility) and indirect consequences (socioeconomic fragility and lack of resilience) of potential hazardous event where the vulnerability factors are described using indicators. This model has a control system which indirectly changes the risk levels by corrective and prospective interventions (e.g. risk identification, risk reduction, disaster management) [39].

\section{Capacity and vulnerability analysis}

In this approach, vulnerabilities are considered as longterm factors that affect a community's ability to respond to events. Currently this model is used by the Federation of the Red Cross and Red Crescent Societies at three areas: physical/material level, including land, climate, environment, health, skills, and labor; social/organizational 
level, including formal political structures and the informal community networks; and motivational/attitudinal area considering how people view themselves and their ability to affect their environment [36].

Vulnerability and risk model: In this model, vulnerability is a hazard-dependent factor caused by three broad and reciprocal factors: Political/institutional factors: These factors are associated with "incommensurate legislation, such as the lack of land-use planning regulations, underdevelopment of the democratic system, incoordination among government institutions, corruption, and lack of instruments to spread financial risks" [39]; Economic factors: Such as the lack of economic resources to implement preventive measures, poverty, and economic dependence; and Sociocultural factors: Such as lack of education, traditional use of land and its management, and deficiencies in social networks to negotiate to raise the level of general welfare [39].

\section{Hazard of place model}

This model, by focusing on the location and general conditions, depicts different elements that contribute to the vulnerability of a particular geographic region. In comparison with "pressure and release" model, this model concentrate on risk-hazard approach and considers risk a product of hazard and vulnerability, and claims that the main reason for disaster occurrence is the vulnerable people's exposure to hazards.

\section{Conclusion}

Although various types of studies have been conducted on the social vulnerability of the elderly, there is no consensus over the concept of social vulnerability and its dimensions, influential elements, and conceptual framework. Consequently, many questions on its concept and methodology have remained unanswered. The lack of consensus on this concept is basically due to the paradigms in quantitative and quantitative investigations. There are two distinct perspectives on social vulnerability assessment. The first perspective relates to the intangible losses and the elements at risk whose material value cannot easily be calculated.

The second perspective includes the underlying socioeconomic factors in a society that causes vulnerability. In this category, methods assess preparedness, coping and adaptive capacity of vulnerability based on the fabric of society. A wide range of factors may be considered as an effective cause of vulnerability, but it should be noted that there is no generally accepted methodology that includes all aspects of social vulnerability [40]. It should be noted that the assessment of social vulnerability based on indicator-based methods is very important and practical.

A review of the literature in this study revealed that the concept of social vulnerability of elderly people to natural disasters has multidimensional, challenging, complex, hidden, and dynamic nature, and has been investigated and assessed from different aspects and by different approaches and contexts. Because of its dynamic nature, various frameworks have been introduced for this concept over time. In this study, it was found out that individual characteristics, economic conditions, culture, and the living place of the elderly are the most important factors affecting their social vulnerability. It causes older people expose to health problems, financial implications, and limitations.

\section{Ethical Considerations}

\section{Compliance with ethical guidelines}

There is no ethical principle to be considered doing this research.

\section{Funding}

This research did not receive any specific grant from funding agencies in the public, commercial, or not-forprofit sectors.

\section{Authors contributions}

All authors have read and approved the manuscript.

\section{Conflict of interest}

The authors declare no conflict of interest.

\section{References}

[1] Khankeh HR, et al. Disaster hospital planing. National Plan. Tehran: University of Social Wellfare and Rehabilitation Sciences; 2012.

[2] Thomas DS, Phillips BD, Lovekamp WE, Fothergill A. Social vulnerability to disasters. Boca Raton, Florida: CRC Press; 2013.

[3] Guha-Sapir D, Hoyois P, Below R. Annual disaster statistical review 2013. Centre for Research on the Epidemiology of Disasters (CRED). Brussels, Belgium: Université catholique de Louvain; 2012. 
[4] Nature. The IUCN Programme 2013-2016 [Internet]. 2012 [Updated 2012 September 4]. Available from: https://cms data.iucn.org/downloads/iucn_programme_2013_2016.pdf

[5] Tourani S, Behzadifar M, Martini M, Aryankhesal A, Mirghaed MT, Salemi M, et al. Health-related quality of life among healthy elderly Iranians: A systematic review and meta-analysis of the literature. Health and Quality of Life Outcomes. 2018; 16(1):18. [DOI:10.1186/s12955-018-0845-7] [PMID] [PMCID]

[6] Chau PH, Gusmano MK, Cheng JO, Cheung SH, Woo J. Social vulnerability index for the older people-Hong Kong and New York city as examples. Journal of Urban Health. 2014; 91(6):104864. [DOI:10.1007/s11524-014-9901-8] [PMID] [PMCID]

[7] Roudi F, Azadi P, Mesgaran M. Iran's population dynamics and demographic window of opportunity. Serra Mall, Stanford: Stanford University; 2017.

[8] Wakui T, Agree EM, Saito T, Kai I. Disaster preparedness among older Japanese adults with long-term care needs and their family caregivers. Disaster Medicine and Public Health Preparedness. 2017; 11(1):31-8. [DOI:10.1017/dmp.2016.53] [PMID]

[9] Evans J. Mapping the vulnerability of older persons to disasters. International Journal of Older People Nursing. 2010; 5(1):63-70. [DOI:10.1111/j.1748-3743.2009.00205.x] [PMID]

[10] Morrow BH. Community resilience: A social justice perspective. Washington: Community \& Regional Resilience Initiative; 2008. [DOI:10.13140/RG.2.1.1278.9604]

[11] Birkmann J. Measuring vulnerability to natural hazards: Toward disaster resilient societies. Tokyo: United Nations University Press; 2006. [PMCID]

[12] Winchester P. Power, choice and vulnerability: A case study in disaster mismanagement in South India. Abingdon: Routledge; 2014. [DOI:10.4324/9781315073590]

[13] Wisner B. Risk reduction indicators. Princeton: TRIAMS Working Paper; 2006.

[14] Parida PK. The social construction of gendered vulnerability to tsunami disaster: The case of coastal Sri Lanka. Journal of Social and Economic Development. 2015; 17(2):200-22. [DOI:10.1007/s40847-015-0019-y]

[15] Stough LM, Kang D. The Sendai framework for disaster risk reduction and persons with disabilities. International Journal of Disaster Risk Science. 2015; 6(2):140-9. [DOI:10.1007/ s13753-015-0051-8]

[16] Birkman, J. Indicators and criteria for measuring vulnerability: Theoretical bases and requirements. Measuring vulnerability to Natural Hazards: Towards Disaster Resilient Societies. Tokyo: United Nations University; 2006.

[17] Rodgers BT, Knafl KA. Integrative literature reviews for the development of concepts: Foundations, techniques and applications. Philadelphia: WB Saunders Company; 2000

[18] Whittemore R, Knafl K. The integrative review: Updated methodology. Journal of Advanced Nursing. 2005; 52(5):54653. [DOI:10.1111/j.1365-2648.2005.03621.x] [PMID]

[19] Tapsell SM, McCarthy S, Faulkner H, Alexander M. Social Vulnerability and Natural Hazards. The Burroughs, London: Iddlesex University; 2010.
[20] Gronlund CJ, Berrocal VJ, White-Newsome JL, Conlon KC, O'Neill MS. Vulnerability to extreme heat by socio-demographic characteristics and area green space among the elderly in Michigan, 1990-2007. Environmental Research. 2015; 136:44961. [DOI:10.1016/j.envres.2014.08.042] [PMID] [PMCID]

[21] Mavhura E, Manyena B, Collins AE. An approach for measuring social vulnerability in context: The case of flood hazards in muzarabani district, zimbabwe. Geoforum. 2017; 86:103-17. [DOI:10.1016/j.geoforum.2017.09.008]

[22] Singh SR, Eghdami MR, Singh S. The concept of social vulnerability: A review from disasters perspectives. International Journal of Interdisciplinary and Multidisciplinary Studies. 2014; 1(6):71-82.

[23] Rhoades JL, Gruber JS, Horton B. Developing an in-depth understanding of elderly adult's vulnerability to climate change. The Gerontologist. 2017; 58(3):567-77.

[24] Cutter SL, Emrich CT, Webb JJ, Morath D. Social vulnerability to climate variability hazards: A review of the literature. Final Report to Oxfam America. 2009; 5:1-44.

[25] Rufat S, Tate E, Burton CG, Maroof AS. Social vulnerability to floods: Review of case studies and implications for measurement. International Journal of Disaster Risk Reduction. 2015; 14:470-86. [DOI:10.1016/j.ijdrr.2015.09.013]

[26] Dunning CM, Durden SE. Social vulnerability analysis: A comparison of tools. Washington: Institute for Water Resources; 2013.

[27] Doppler JV. Gender and Tsunami (Doctoral dissertation, uniwien) [MSc. thesis]. Vienna: University of Vienna; 2009.

[28] Barusch AS. Disaster, vulnerability, and older adults: Toward a social work response. Journal of Gerontological Social Work. 2011; 54(4):347-50. [DOI:10.1080/01634372.2011.58282] [PMID]

[29] Kim H, Zakour M. Disaster preparedness among older adults: Social support, community participation, and demographic characteristics. Journal of Social Service Research. 2017; 43(4):498-509. [DOI:10.1080/01488376.2017.1321081]

[30] Guillard-Gonçalves C, Cutter SL, Emrich CT, Zêzere JL. Application of Social Vulnerability Index (SoVI) and delineation of natural risk zones in Greater Lisbon, Portugal. Journal of Risk Research. 2015; 18(5):651-74.

[31] Marshall Jr, Mathews S. Disaster preparedness for the elderly: An analysis of international literature using symbolic interactionist perspective. The Journal of the Economics of Ageing. 2010; 2(2):79-92.

[32] Paul SK. Vulnerability concepts and its application in various fields: A review on geographical perspective. Journal of Life and Earth Science. 2014; 8(2013):63-81. [DOI:10.3329/ jles.v8i0.20150]

[33] Bakhshipour RA, Peyrovi H, Abedian A. [Investigating relationship between satisfaction with life and social support with mental health among freshman students of Tehran university (Persian)]. The Quarterly Journal of Fundamentals of Mental Health. 2006; 7(27-28):145-52

[34] Alexander D. Models of social vulnerability to disasters. Revista Crítica de Ciências Sociais. 2012; 4(4):21-40.

[35] Jogia J, Kulatunga U, Yates GP, Wedawatta G. Culture and the psychological impacts of natural disasters: Implications 
for disaster management and disaster mental health. Built and Human Environment Review. 2014; 7(1):1.

[36] Leon D, Villagran JC. Vulnerability: A conceptual and methodological review institute of environment and human security. Tokyo: United Nations University Press; 2006.

[37] Fernandez LS, Byard D, Lin CC, Benson S, Barbera JA. Frail elderly as disaster victims: Emergency management strategies. Prehospital and Disaster Medicine. 2002; 17(2):67-74. [DOI:10.1017/S1049023X00000200] [PMID]

[38] Ciurean RL, Schroter D, Glade T. Conceptual frameworks of vulnerability assessments for natural disasters reduction. InApproaches to disaster management. London: IntechOpen; 2017. [DOI: $10.5772 / 55538]$

[39] Soltani Nejad A, Barshan A, Baniasad A, Soltani Nejad A, Sam A, Sadie A. [Investigating social vulnerability of the elderly in the earthquakes of Bam, Varzaghan, and Ahar (Persian)]. Iranian Journal of Ageing, 2017; 12(3):360-371. [DOI:10.21859/sija.12.3.360]

[40] Gautam D. Assessment of social vulnerability to natural hazards in Nepal. Natural Hazards and Earth System Sciences, 2017; 17(12):2313-20. [DOI:10.5194/nhess-17-2313-2017] 